\title{
Editorial: Structured Edible Oil: Towards a New Generation of Fat Mimetics
}

\author{
Miguel A. Cerqueira ${ }^{1 *}$, Luiz H. Fasolin ${ }^{2 *}$ and Stephen R. Euston ${ }^{3 *}$ \\ ${ }^{1}$ International Iberian Nanotechnology Laboratory, Braga, Portugal, ${ }^{2}$ Department of Food Engineering, Faculty of Food \\ Engineering, Campinas State University, Campinas, Brazil, ${ }^{3}$ School of Engineering and Physical Sciences, Institute of \\ Biological Chemistry, Biophysics and Bioengineering, Heriot-Watt University, Edinburgh, United Kingdom
}

Keywords: oleogel, organogel, oleofoam, emulsion, fat mimetic

\section{Editorial on the Research Topic}

\section{OPEN ACCESS}

Edited by:

Guillermo Raul Castro,

Consejo Nacional de Investigaciones

Científicas y Técnicas

(CONICET), Argentina

Reviewed by:

Suzana Ferreira-Dias,

Instituto Superior Agronomia

Universidade de Lisboa, Portugal Guadalupe Virginia Nevárez-Moorillón,

Autonomous University of

Chihuahua, Mexico

*Correspondence:

Miguel A. Cerqueira

miguel.cerqueira@inl.int

Luiz H. Fasolin

Ifasolin@unicamp.br

Stephen R. Euston

s.r.euston@hw.ac.uk

Specialty section:

This article was submitted to

Sustainable Food Processing,

a section of the journal

Frontiers in Sustainable Food Systems

Received: 12 January 2021

Accepted: 25 February 2021

Published: 26 March 2021

Citation:

Cerqueira MA, Fasolin LH and Euston SR (2021) Editorial: Structured Edible Oil: Towards a New Generation of Fat Mimetics.

Front. Sustain. Food Syst. 5:652644. doi: 10.3389/fsufs.2021.652644

\section{Structured Edible Oil: Towards a New Generation of Fat Mimetics}

Saturated and trans (produced during partial hydrogenation of unsaturated oils) fatty acids in food are related to several comorbidities and mainly to the raise in blood cholesterol levels, which in turn is a risk factor for cardiovascular disease (CVD), and as such, hydrogenated fats have become one of the biggest health issues around processed foods in recent years. Not only does this contribute to increased morbidity but is also a major economic cost for national healthcare providers and governments. The European Heart Network in the European Cardiovascular Disease Statistics (Wilkins et al., 2017) put the total costs of CVD (healthcare and other-productivity loss, etc.) as $£ 26 \mathrm{Bn}$ in 2015 alone. The cost of CVD to the UK is around $€ 14.82 \mathrm{Bn}$ per year (Public Health England, 2017).

Some of the blame for dietary related health issues has been leveled at the food industry for producing high calorie, energy dense foods (Wilding, 2012). Wilding (2012) has described the food industry as "an unhealthy alliance of producers and marketers," which he says have "successfully promoted energy dense foods, many of which provide positive reinforcement that increases consumption, effectively producing a "cafeteria diet" for the whole human population-a well-proved way of causing obesity in experimental animals."

In their defense, manufacturers have tried introducing reduced calorie foods, where fats (or carbohydrates) have been removed and replaced with other ingredients to lower the energy density. Take up of these has been low, however, as their taste and texture are often considered inferior by the consumer (Hamilton et al., 2000; McEwan and Sharp, 2000). Poole et al. (2020) have pointed out that even the smallest change to texture and flavor of a food product can disenfranchise the consumer base, which is no incentive for manufacturers to reformulate their products. Clearly, if current strategies for fat replacement or low energy density foods production are not producing products of sufficient quality, then other strategies for the formulation of healthier foods should be researched. The challenges are mainly related to the physicochemical properties of fats and their influence on food structure and organoleptic properties. Therefore, the scientific community and the industry are looking for new ways to structure oils and replace the existing fats by healthier fats and obtain reduced low-fat content products while maintaining the organoleptic properties and convenience of the products, ensuring acceptance by consumers.

In this Research Topic Structured Edible Oil: Towards a New Generation of Fat Mimetics emerging methods for the replacement of fats in food systems are presented. These are focused on ways to structure polyunsaturated edible oils (so-called oleogelation) so they can be used in place of their saturated counterparts. A range of oleogelation mechanisms are explored. Avendaño-Vásquez et al., studied the well-known ability of alkyl ester waxes to self-associate in oils to form an oleogel, 
demonstrating that it is possible to tune the melting properties of the gel by varying the relative chain lengths of the alkyl chains and through the addition of the monoacylglycerol 1-stearoylglycerol. In several of the studies, the use of non-lipophilic gelators is demonstrated. Bascuas et al. use the hydrocolloids hydroxymethyl cellulose and xanthan gum with the emulsion template method to produce oleogels with a high oxidative and physical stability. Gallegos Soto et al. have taken the use of hydrocolloids a step further and demonstrate that use chitosan in combination with anionic polysaccharides to replace $80 \%$ of the cocoa butter in dark chocolate with oleogelled oils. A third and novel type of oleogelator reported here by Whitby is the use of fumed silica particles. These are able to aggregate into branched fractal structures that form a network gel in the oil, with the structure and properties making them well-suited to encapsulation of bioactive ingredients in functional foods. The remaining papers review various aspects for oleogel structure and applications. Park and Maleky give a mini-review concentrating on food applications and in particular the nutritional, texture, and stability of various oleogels in real formulations. Fameau et al. report a very interesting study on oleofoams, oil systems where air bubble are trapped in an oleogelled network. They highlight the potential of using these to replace solid fat with liquid oils and

\section{REFERENCES}

Hamilton, J., Knox, B., Hill, D., and Parr, H. (2000). Reduced fat productsconsumer perceptions and preferences. Br. Food J. 102, 494-506. doi: 10.1108/00070700010336454

McEwan, J. A., and Sharp, T. M. (2000). Technical, economic and consumer barriers to the consumption of reduced fat bakery products. Nutr. Food Sci. 30, 16-18. doi: 10.1108/00346650010304710

Poole, J., Bentley, J., Barraud, L., Samish, I., Dalkas, G., Matheson, A., et al. (2020). Rising to the challenges: Solution-based case studies highlighting innovation and evolution in reformulation. Nutr. Bull. 45, 332-340. doi: $10.1111 /$ nbu. 12456

Public Health England (2017). Cardiovascular Disease Prevention Action Plan - 2017 to 2018. Available online at: https://assets.publishing.service. gov.uk/government/uploads/system/uploads/attachment_data/file/648190/ cardiovascular_disease_prevention_action_plan_2017_to_2018.pdf (accessed 11 January 2021). air bubbles, and point to the very high stability of the air bubbles with adsorbed gelators as being a key attribute of these systems. Finally, Wijarnprecha et al. discuss the highly important topic of oleogel water-in-oil emulsions. Some oleogels, particularly those containing gelators that form hydrates, are highly unstable in the presence of even small amounts of water. Solving this is the key to opening up oleogel technology to a wide range of intermediate and high-water activity foods, including food emulsions.

Fat mimetics will be for sure a trend topic in the next 5-10 years, and it is clear from the papers published in this Research Topic the great possibilities of using oleogelation in this regard. The topics covered represent some of the infinite possibilities for research and innovation in this field. The work that is still ongoing foresees great endeavors in this area and it is expected that industry will adopt some of the new ideas for their products.

For this Research topic, it is important to highlight the important role of our friend and colleague Dr. Ashok Patel who served as a Topic Editor and who sadly passed away in May 2020.

\section{AUTHOR CONTRIBUTIONS}

All authors listed have made a substantial, direct and intellectual contribution to the work, and approved it for publication.

Wilding, J. (2012). Are the causes of obesity primarily environmental? Yes. BMJ 354:e5843. doi: 10.1136/bmj.e5843

Wilkins, E., Wilson, L., Wickramasinghe, K., Bhatnagar, P., Leal, J., Luengo-Fernandez, R., et al. (2017). European Cardiovascular Disease Statistics 2017. Brussels: European Heart Network.

Conflict of Interest: The authors declare that the research was conducted in the absence of any commercial or financial relationships that could be construed as a potential conflict of interest.

Copyright (c) 2021 Cerqueira, Fasolin and Euston. This is an open-access article distributed under the terms of the Creative Commons Attribution License (CC BY). The use, distribution or reproduction in other forums is permitted, provided the original author(s) and the copyright owner(s) are credited and that the original publication in this journal is cited, in accordance with accepted academic practice. No use, distribution or reproduction is permitted which does not comply with these terms. 